This item was submitted to Loughborough's Research Repository by the author.

Items in Figshare are protected by copyright, with all rights reserved, unless otherwise indicated.

\title{
Two-soliton interaction as an elementary act of soliton turbulence in integrable systems
}

PLEASE CITE THE PUBLISHED VERSION

http://dx.doi.org/10.1016/j.physleta.2012.11.037

\section{PUBLISHER}

(C) Elsevier

VERSION

AM (Accepted Manuscript)

\section{PUBLISHER STATEMENT}

This work is made available according to the conditions of the Creative Commons Attribution-NonCommercialNoDerivatives 4.0 International (CC BY-NC-ND 4.0) licence. Full details of this licence are available at: https://creativecommons.org/licenses/by-nc-nd/4.0/

\section{LICENCE}

CC BY-NC-ND 4.0

\section{REPOSITORY RECORD}

Pelinovsky, Efim N., E.G. Shurgalina, A.V. Sergeeva, Tatiana G. Talipova, G.A. El, and Roger H.J. Grimshaw. 2019. "Two-soliton Interaction as an Elementary Act of Soliton Turbulence in Integrable Systems". figshare. https://hdl.handle.net/2134/16948. 


\section{Two-soliton interaction as an elementary act of soliton turbulence in integrable systems}

E.N. Pelinovsky ${ }^{1,2}$, E.G. Shurgalina ${ }^{2}$, A.V. Sergeeva ${ }^{1}$, T.G. Talipova ${ }^{1}$, G.A. $\mathrm{El}^{3 *}$ and R.H.J. Grimshaw ${ }^{3}$

${ }^{1}$ Department of Nonlinear Geophysical Processes, Institute of Applied Physics, Russian Academy of Sciences, Nizhny Novgorod, Russia

2 Department of Applied Mathematics, Nizhny Novgorod Technical University, Nizhny Novgorod, Russia

${ }^{3}$ Department of Mathematical Sciences, Loughborough University, UK

${ }^{*}$ Corresponding author. Tel: +44 1509 222869; Fax: +44 1509 223969; e-mail: g.el@lboro.ac.uk 


\begin{abstract}
Two-soliton interactions play a definitive role in the formation of the structure of soliton turbulence in integrable systems. To quantify the contribution of these interactions to the dynamical and statistical characteristics of the nonlinear wave field of soliton turbulence we study properties of the spatial moments of the two-soliton solution of the Korteweg - de Vries (KdV) equation. While the first two moments are integrals of the KdV evolution, the third and fourth moments undergo significant variations in the dominant interaction region, which could have strong effect on the values of the skewness and kurtosis in soliton turbulence.
\end{abstract}

Keywords: KdV equation, soliton, turbulence

\title{
1 Introduction
}

Solitons represent an intrinsic part of nonlinear wave field in weakly dispersive media and their deterministic dynamics in the framework of the Korteweg- de Vries (KdV) equation is understood very well (see e.g. $[1,2,3]$ ). At the same time, description of statistical properties of a random ensemble of solitons (or a more general problem of the KdV evolution of a random wave field) still remains to a large extent an unsolved problem, especially in the context of concrete physical applications. In particular, importance of this problem for the description of wind-generated waves on shallow water was demonstrated in [4] $-[9]$. From the theoretical point of view the description of a random soliton wave field is complementary to the "integrable wave turbulence" theory outlined in a recent paper by Zakharov [10].

The macroscopic dynamics of random soliton ensembles (soliton gases) in integrable systems are determined by the fundamental "microscopic" properties of soliton interactions: (i) soliton collisions are elastic, i.e. the interaction does not change the soliton amplitudes (or, more precisely, the discrete spectrum levels in the associated linear spectral problem); (ii) after the interaction, each soliton gets an additional phase shift; (iii) the total phase shift of a 'trial' soliton acquired during a certain time interval can be calculated as a sum of the "elementary" phase shifts in pairwise collisions of this soliton with other solitons during this time interval. Thus the dynamics of a soliton gas are essentially determined by two-soliton interactions.

The study of soliton gases was initiated by Zakharov in [11] where an approximate kinetic equation for random KdV solitons when their spatial density is small was der. This equation describes spatio-temporal evolution of the distribution function of solitons over the (IST) spectrum. The full kinetic equation for the KdV soliton gas of arbitrary density was derived in [12] (see also [13]) using the thermodynamic limit of the Whitham modulation equations and then was generalised in [14] to other integrable systems. The kinetic description of a soliton gas makes an emphasis on the particle-like nature of solitons. At the same time, solitons represent nonlinear coherent wave structures so the total random nonlinear wave field associated with a soliton gas can be naturally interpreted as soliton turbulence [15]. In view of the outlined definitive role of two-soliton interactions, it is natural to ask: what is their specific (qualitative and quantitative) contribution to the statistical properties of soliton turbulence? In classical (both hydrodynamic and wave) turbulence theories the random field properties are usually described in terms of statistical moments (see e.g. [16], [17]). This provides one with a natural motivation to start with the study of the properties of the spatial 
moments of the two-soliton $\mathrm{KdV}$ solution. In spite of the elementary nature of this problem it has apparently never been considered before. In the context of the soliton turbulence description, the knowledge of "primitive" dynamics of the spatial moments of two-soliton solutions is a necessary ingredient in the understanding of the behaviour of the statistical moments of the random KdV wave field.

Since the first and the second spatial moments of the two-soliton solution are conserved under the KdV evolution, our main focus in this Letter will be on the properties of the third and fourth moments which vary with time and which, after appropriate ensemble averaging, will affect the behaviour of the skewness and kurtosis of the probability distribution of the random wave field in the $\mathrm{KdV}$ soliton turbulence. These two statistical characteristics are also known to play important role in the theory of rogue waves [18].

\section{Dynamics of two-soliton interactions}

Although multisoliton solutions of the $\mathrm{KdV}$ equation had been known since the very beginning of the soliton theory creation [19], [20], the nature of the mass/momentum/energy exchange occurring during the interaction of two solitons have been continued to be the subject of rather active study (see [21] and references therein). In view of the outlined in the Introduction key role of the two-soliton interactions in the formation of the structure of soliton turbulence we shall need to briefly revisit here some of their basic properties.

We shall use the canonical form of the KdV equation

$$
u_{t}+6 u u_{x}+u_{x x x}=0
$$

The two-soliton solution of (1) has the form (see e.g. [2], [3])

$$
\begin{aligned}
& u_{2}(x, t)=2 \partial_{x}^{2} \ln [\tau(x, t)], \\
& \text { where } \quad \tau=1+e^{\phi_{1}}+e^{\phi_{1}}+\alpha^{2} e^{\phi_{1}+\phi_{2}} \text {, } \\
& \alpha=\frac{\eta_{2}-\eta_{1}}{\eta_{1}+\eta_{2}}, \quad \phi_{i}=-2\left(\eta_{i} x-4 \eta_{i}^{3} t-\xi_{i}\right), \quad i=1,2 .
\end{aligned}
$$

Here $-\eta_{1,2}^{2}$ are the discrete spectrum points in the associated IST formalism and $\xi_{i}$ are the initial phases of solitons. When $t \gg 1$ solution (2) asymptotically (up to exponentially small terms) transforms into a superposition of two single-soliton solutions (see e.g. [1], [3]):

$$
u_{2} \sim A_{1} \operatorname{sech}^{2}\left[\eta_{1} x-4 \eta_{1}^{3} t-\xi_{1}-\Delta_{1}\right]+A_{2} \operatorname{sech}^{2}\left[\eta_{2} x-4 \eta_{2}^{3} t-\xi_{2}-\Delta_{2}\right]
$$

where the amplitudes $A_{i}=2 \eta_{i}^{2}, i=1,2$ and the phase shifts $\Delta_{1,2}$ of the solitons due to the interaction are: $\Delta_{1,2}= \pm \ln |\alpha|$ assuming $A_{1}>A_{2}$. We note that two-soliton KdV solution (2) can be represented in a number of equivalent forms emphasising different aspects of the soliton interaction dynamics (see e.g. [21]).

Let at the initial moment the taller soliton with amplitude $A_{1}$ be located behind the shorter one with the amplitude $A_{2}$. Since the $\mathrm{KdV}$ soliton speed is proportional to its amplitude, the first soliton will catch up the second one and the nonlinear interaction will take place within certain space-time "dominant interaction region" (see [3]). There are three types of the behaviour in the dominant interaction region depending on the amplitude ratio $r=A_{1} / A_{2}>1$ of the interacting solitons [22]: 
(i) if $1<r<\frac{3+\sqrt{5}}{2} \approx 2.62$, then the interacting solitons interchange their roles without passing through each other. They never "stick together" into a single unimodal pulse and always retain their "identity" during the interaction. This type of interaction is often called the "exchange interaction". At the moment when the strength of the interaction reaches its peak the double wave assumes a symmetric two-hump profile with the local minimum $u=u^{*}=A_{1}-A_{2}$ at the centre (see e.g. [23]).

(ii) if $\frac{3+\sqrt{5}}{2}<r<3$ the nature of the interaction changes so that the taller soliton first absorbs the shorter one and then re-emits it. Similar to the case (i) the solitons never merge into a single hump, but at the same time the double wave never assumes a symmetric shape. This scenario can be associated with the transition from the "exchange" to the "overtaking interaction". The amplitude of the shorter soliton grows during the absorbtion phase and assumes its maximum value $\frac{2}{5}\left[A_{1}+A_{2}+\left(A_{1}^{2}+A_{2}^{2}-3 A_{1} A_{2}\right)^{1 / 2}\right]$ at the moment of the strongest interaction, say $t=t^{*}$. At the same moment $t=t^{*}$ the value of the double wave amplitude reaches its minimum $u_{m}=\min \left[\max \left\{u_{2}(x, t)\right\}: t>0\right]$.

(iii) if $r>3$, then the soliton interaction mechanism is essentially the same as in case (ii) but now the solitons merge into a single unimodal hump in the dominant interaction region, before they separate again. This scenario is usually associated with the "overtaking interaction". The minimum of the resulting single pulse amplitude achieved at the moment of the strongest interaction is $u_{m}=A_{1}-A_{2}$.

In all three above-mentioned scenarios, the resulting double wave in the dominant interaction region is wider than each of the interacting solitons and has a smaller amplitude than that of the taller soliton before the interaction. One can derive an ordinary differential equation describing the exact dynamics of the local maxima of the 2-soliton solution (see [22]). However, for our purposes it is sufficient to present a simple plot of the value of the double wave minimal amplitude $u_{m}$ defined above, versus the amplitude ratio $r^{-1}=A_{2} / A_{1}$ of the individual interacting solitons. The plot of $u_{m}\left(A_{2} / A_{1}\right)$ obtained from direct numerical simulations of the collisions of different pairs of the $\mathrm{KdV}$ solitons is presented in Fig. 1. It was assumed in the simulations that the initial amplitude of the taller soliton $A_{1}=1$.

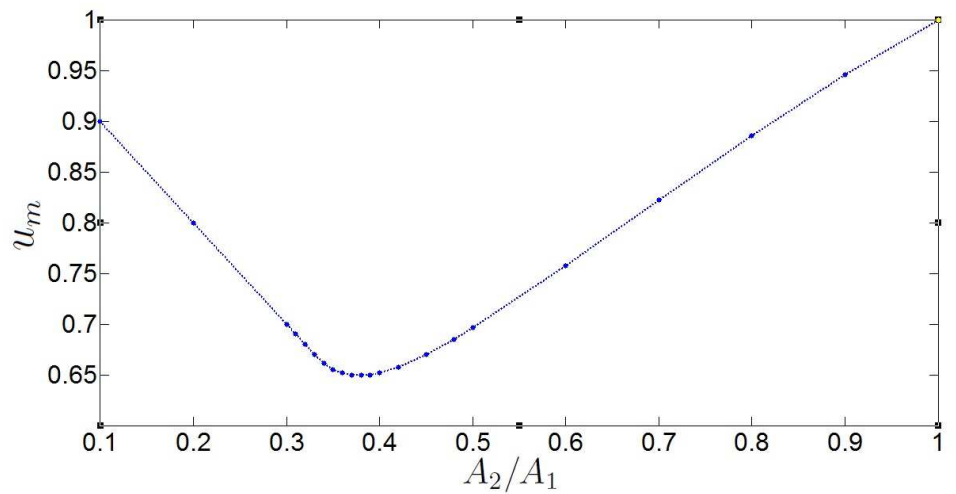

Figure 1: Dependence of the minimum of the double wave amplitude $u_{m}=$ $\min \left[\max \left\{u_{2}(x, t)\right\}: t>0\right]$ on the soliton amplitude ratio $A_{2} / A_{1}$ in the two-soliton solution.

As one can see, the absolute minimum of the function $u_{m}$ is achieved at $A_{2} / A_{1}=1 / 2.62 \approx$ 0.38 , which is the upper boundary of the transition interval $0.33<A_{2} / A_{1}<0.38$ between the 
exchange and overtaking soliton interaction scenarios (see previous Section). This property of the two-soliton KdV solutions could have important implications for the analysis of the random soliton wave field, in particular, for establishing the relation between the distribution of the values of local extrema in the soliton turbulence and its spectral (IST) composition (we recall that the initial soliton amplitudes $A_{1,2}$ are directly related to the IST spectrum - see $(2))$.

\section{Effect of soliton interactions on the integral charac- teristics of the wave field}

Most of the features of the two-soliton interaction described in the previous section are known very well. However, the effect of the soliton interaction on the integral characteristics of the wave field to the best of our knowledge had not been considered before. It is this effect that is of our primary concern in this Letter since it will have direct implications for the theory of the KdV soliton turbulence.

As is known, the KdV equation has an infinite number of conserved quantities (Kruskal integrals) (see e.g. $[1,2,3]$ ); below we present the first four of them:

$$
\begin{aligned}
& I_{1}=\int_{-\infty}^{\infty} u(x, t) d x, \\
& I_{2}=\int_{-\infty}^{\infty} u^{2}(x, t) d x \\
& I_{3}=\int_{-\infty}^{\infty}\left[u^{3}-\frac{1}{2} u_{x}^{2}\right] d x, \\
& I_{4}=\int_{-\infty}^{\infty}\left[u^{4}-2 u u_{x}^{2}+\frac{1}{5}\left(u_{x x}\right)^{2}\right] d x .
\end{aligned}
$$

The first three integrals $(4-6)$ are usually associated with the "mass", "momentum" and "energy" conservation although they do not necessarily have physical meaning of the corresponding physical entities. All Kruskal integrals are conserved under the KdV evolution (assuming vanishing at infinity or periodic boundary conditions for the wave field) so it is clear from the very begining that these quantities are not affected by the soliton interaction. Nevertheless, it is interesting to know their dependence on the soliton amplitudes since the higher integrals (starting from the 3rd) are not necessarily positive definite. Formally, one would need to use full two-soliton solution $(2)$ in $(4-7)$ but the calculation can be dramatically simplified in view of the conservation of $I_{1}, I_{2}, I_{3}, I_{4}$, so that one can use asymptotic expression (3) instead of the full solution (2) and all the integrals can be evaluated for each soliton separately. As a result, after somewhat lengthy calculation, we obtain:

$$
\begin{aligned}
& I_{1}=4\left(\eta_{1}+\eta_{2}\right)=2 \sqrt{2}\left(A_{1}^{1 / 2}+A_{2}^{1 / 2}\right), \\
& I_{2}=\frac{16}{3}\left(\eta_{1}^{3}+\eta_{2}^{3}\right)=\frac{4 \sqrt{2}}{3}\left(A_{1}^{3 / 2}+A_{2}^{3 / 2}\right), \\
& I_{3}=\frac{32}{5}\left(\eta_{1}^{5}+\eta_{2}^{5}\right)=\frac{4 \sqrt{2}}{5}\left(A_{1}^{5 / 2}+A_{2}^{5 / 2}\right), \\
& I_{4}=\frac{256}{35}\left(\eta_{1}^{7}+\eta_{2}^{7}\right)=\frac{16 \sqrt{2}}{35}\left(A_{1}^{7 / 2}+A_{2}^{7 / 2}\right) .
\end{aligned}
$$


Remarkably, all the integrals $I_{1}, I_{2}, I_{3}, I_{4}$ turn out to be positive definite so, taking into account the long-time asymptotic representation of the $N$-soliton solution as the sum of individual solitons, analogous to (3), one can conclude that their values increase as the number of solitons increases. As one could expect, the "higher" integrals have stronger dependence on the amplitude than the "lower" ones.

In turbulence theory one is usually interested in the standard moments of the form

$$
M_{n}(t)=\int_{-\infty}^{\infty} u^{n}(x, t) d x, \quad n=1,2,3, \ldots
$$

Obviously, for the two-soliton solution the first two moments (12) $M_{1}$ and $M_{2}$ coincide with the respective Kruskal integrals $I_{1}$ and $I_{2}$ and, therefore, are conserved. In turbulence theory $M_{1}$ and $M_{2}$ define the mean value and variance of the random wave field respectively, and their constancy means that nonlinear interactions do not affect these two important parameters (we note that in many problems nonlinearity leads to variations of the mean, e.g. in the so-called wave setup phenomenon in fluid dynamics).

The next two moments, $M_{3}(t)$ and $M_{4}(t)$, are related to the skewness and kurtosis of the probability distribution of the turbulent field. They do not coincide with the Kruskal integrals $I_{3}$ and $I_{4}$ so one should not expect that they will be conserved in soliton turbulence. Numerical evaluation of $M_{3}$ and $M_{4}$ for the two-soliton solution (2) with $A_{1}=1$ and $A_{2}=0.3$ shows that these moments decrease in the dominant interaction region (see Fig. 2). Outside

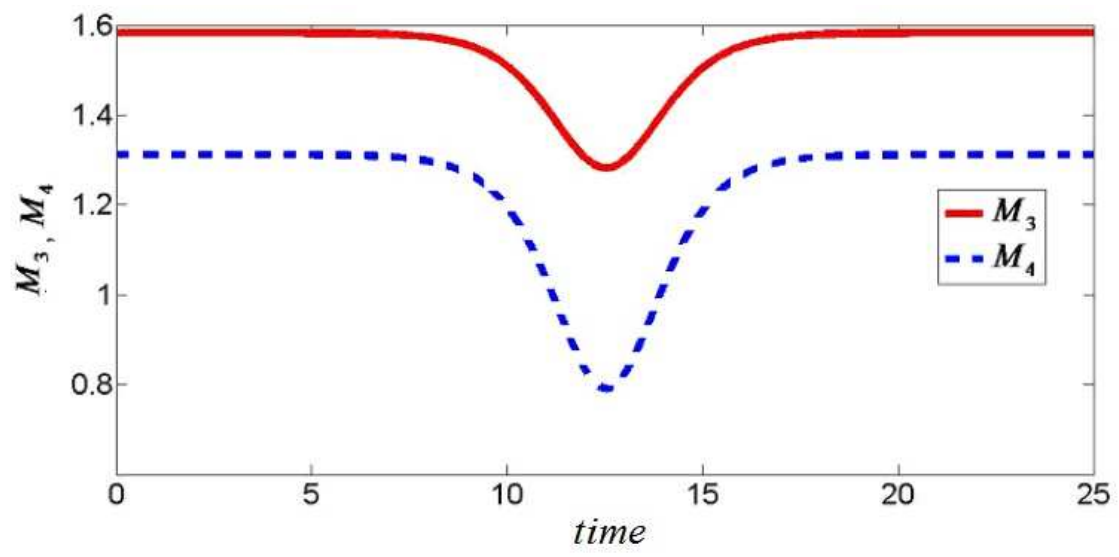

Figure 2: The time dependence of the moments $M_{3}, M_{4}$ in the two-soliton interaction with $A_{1}=1, A_{2}=0.3$

the interaction region $M_{3}$ and $M_{4}$ assume the values corresponding to the superposition of non-interacting solitons (3):

$$
\begin{aligned}
& M_{3}^{0}=\frac{8 \cdot 16}{15}\left(\eta_{1}^{5}+\eta_{2}^{5}\right)=\frac{16 \sqrt{2}}{15}\left(A_{1}^{5 / 2}+A_{2}^{5 / 2}\right), \\
& M_{4}^{0}=\frac{16 \cdot 32}{35}\left(\eta_{1}^{7}+\eta_{2}^{7}\right)=\frac{32 \sqrt{2}}{35}\left(A_{1}^{7 / 2}+A_{2}^{7 / 2}\right) .
\end{aligned}
$$

One can see that the variations of the 3rd and 4th moments are quite significant (up to $30 \%$ ) which implies that soliton interactions can strongly affect the higher moments of the wave 
field, while the 1st and the 2nd moments remain unaffected. Physically, the decrease of the 3rd and 4th moments due to soliton interactions can be explained by the above-mentioned decrease of the resulting pulse amplitude during the interaction. Also, as one can see from the conservation of the third Kruskal integral (6), the decrease of the third moment $\int u^{3} d x$ results in the decrease of the integral $\int\left(u_{x}\right)^{2} d x$ which implies smoothing of the monotone slopes of the pulse during the interaction. Our simulations of two-soliton collisions characterised by different values of the definitive interaction parameter $r=A_{1} / A_{2}$ show the same qualitative behaviour of the higher moments in the dominant interaction region, while the amplitude of their variations depends on the value of $r$. In Fig. 3 we present the numerical results for the amplitudes of the relative variations, $\Delta M_{i} / M_{i}^{0}$, where $\Delta M_{i}=M_{i}^{0}-M_{i}^{(m i n)}, i=3$, 4 , versus $r^{-1}=A_{2} / A_{1}$. Again, in our numerical simulations we have assumed that the amplitude of the greater soliton $A_{1}=1$. Both curves are nonmonotone and have their extremum (maximum) at the same value of the amplitude ratio $A_{2} / A_{1} \approx 0.32$ which is close to the lower boundary of the transition region $0.33<A_{2} / A_{1}<0.38$ separating the exchange and overtaking scenarios of the two-soliton interaction. Thus the two-soliton interactions with the amplitude ratio in the transition interval are expected to have greater impact on the higher moments in soliton turbulence.

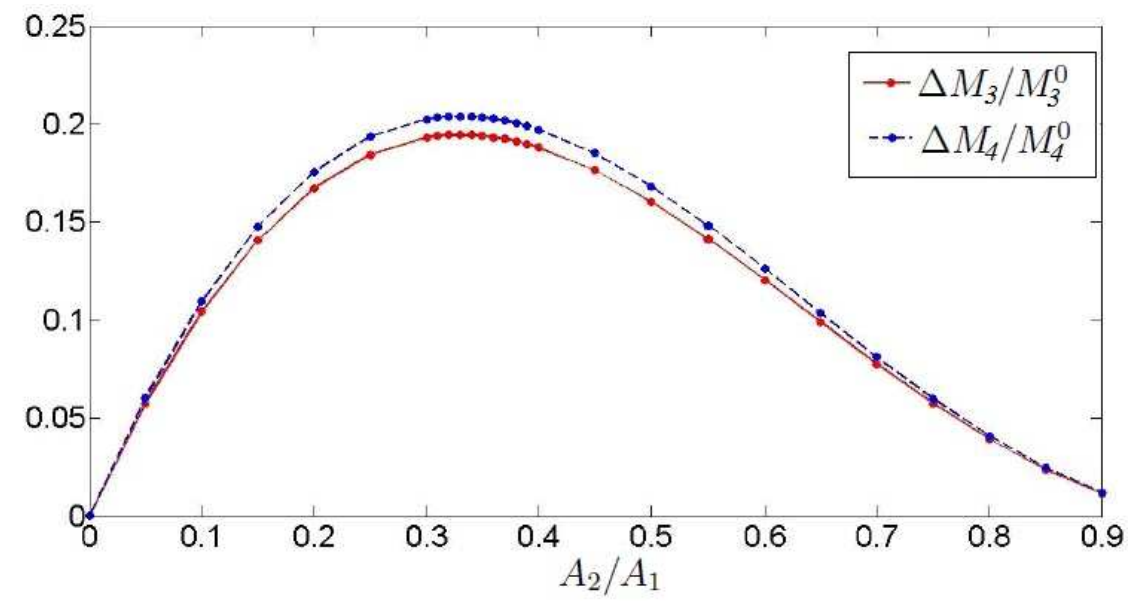

Figure 3: Dependence of the relative variations $\Delta M_{i} / M_{i}^{0}$ of the third and fourth moments of the two-soliton solution on the soliton amplitude ratio $A_{2} / A_{1}$.

To the best of our knowledge, the described effect of soliton interactions on the higher moments of multisoliton solutions has never been reported in the literature. Taking into account the key role of the higher moments in the characterisation of the skewness and kurtosis of the turbulent field, an immediate implication of this effect in the context of soliton turbulence is that the pairwise interactions of solitons must decrease the skewness and kurtosis (compared to their values for the gas of noninteracting solitons). It is clear the quantitative contribution of this effect will depend on the density of the soliton gas (frequency of soliton collisions) and on its spectral (IST) composition (the ratios $A_{1} / A_{2}$ involved), i.e. on the spectral distribution function of the soliton gas [14]. Thus, for inhomogeneous soliton turbulence, when the density of solitons depends on the spatial coordinate, the analysis of the higher statistical moments behaviour will be coupled with the kinetic description of the associated soliton gas. 
In conclusion of this section we note that in classical and wave turbulence theories, along with spatial moments, one is also interested in the Fourier transform of the velocity field, its power spectrum etc. A similar description can be introduced for soliton turbulence as well. The description of the Fourier spectrum evolution in the multisoliton solutions of integrable systems is also directly related to spectral algorithms of the numerical simulations of emergence, propagation and interaction of solitons in nonlinear dispersive media (see e.g. $[24])$.

\section{Conclusions}

We have shown that the two-soliton interaction in the framework of the KdV equation leads to the decrease of the $3 \mathrm{rd}$ and $4 \mathrm{rth}$ moments $M_{3,4}$ of the nonlinear wave field while the 1st and the 2nd moments remain unchanged due to the conservation of the mass and momentum. The magnitudes of the relative variations of $M_{3}, M_{4}$ turn out to be nonmonotone functions of the soliton amplitude ratio $A_{2} / A_{1}$ each having a single maximum located at the point $A_{2} / A_{1} \approx 0.32$, close the boundary of the transition region between the exchange and overtaking scenarios of two-soliton interactions. The qualitative implication of this dynamical effect for the soliton turbulence theory will be a decrease of the skewness and kurtosis of the turbulent wave field in the regions of higher density of solitons. The quantitative analysis of the effect of soliton interactions on the structure of soliton turbulence will be made in our future publications.

\section{Acknowledgements}

This work was supported by grants from the Volkswagen Foundation (EP and AS), Federal Target Programme "Research and scientific-pedagogical cadres of Innovative Russia" for 2009-2013 (EP), MK-4378.2011.5 (AC), RFBR 12-05-00472 (TT) and 11-05-00216 (EP and ES), Dynastiya and MK-1440.2012.5 (ES), the Royal Society of London (EP and RG).

\section{References}

[1] S.P. Novikov, S.V. Manakov, L.P. Pitaevskii, and V.E Zakharov, The Theory of Solitons: The Inverse Scattering Method. Consultants, New York (1984).

[2] A.C. Newell, Solitons in mathematics and physics. SIAM, Philadelphia (1985)

[3] P.G. Drazin and R.S. Johnson Solitons: an introduction. Cambridge University Press (1993).

[4] A.R. Osborne, Phys. Rev. Lett. 71 (1993) 3115-3118.

[5] A.R. Osborne, Phys. Rev. E, 52 (1995) 1105-1122.

[6] A.R. Osborne, E. Segre and G. Boffetta, Phys. Rev. Lett. 67 (1991) 592-595.

[7] A.R. Osborne, M. Serio, L. Bergamasco and L. Cavaleri, Physica D 123 (1998) 64-81 
[8] M. Brocchini, R. Gentile, Continental Shelf Research, 21 (2001) 1533-1550

[9] E. Pelinovsky and A. Sergeeva (Kokorina), Eur. J. Mech., 25 (2006) 425-434

[10] V.E. Zakharov, Stud. Appl. Math. 122 (2009) 219 -234.

[11] V.E. Zakharov, Sov. Phys. JETP, 33 (1971) 538-541

[12] G.A. El, Phys. Lett. A 311 (2003) 374 - 383.

[13] G.A. El, A.M. Kamchatnov, M.V. Pavlov, S.A. Zykov, J. Nonl. Sci. 21 (2011) 151-191

[14] G.A. El and A.M. Kamchatnov, 95 (2005) Art. No 204101

[15] G.A. El, A.L. Krylov, S.A. Molchanov, S. Venakides, Advances in Nonlinear Mathematics and Science, Physica D 152-153 (2001) 653-664.

[16] A.S. Monin, A.M. Yaglom, Statistical fluid mechanics, Volume I, Dover Pubilications (2007) $784 \mathrm{p}$.

[17] S. Nazarenko, Wave turbulence, Springer (2011) 279 p.

[18] Ch. Kharif, E. Pelinovsky and A. Slunyaev, Rogue Waves in the Ocean, Springer (2009) $216 \mathrm{p}$.

[19] N. J. Zabusky, M. D. Kruskal, Phys. Rev. Lett. 15 (1965) 240243.

[20] C. S. Gardner, J.M. Greene, M. D. Kruskal, R. M. Miura, Phys. Rev. Lett. 19 (1967) 10951097.

[21] N. Benes, A. Kasman, and K. Young, J. Nonl. Sci., 16 (2006) 179-200.

[22] P. D. Lax, Commun. Pure Appl. Math. 21 (1968), 467490.

[23] A.V. Slyunyaev and E.N. Pelinovsky, J. Exp. Theor. Phys. 89 (1999) 173 - 181

[24] A. Salupere, The pseudospectral method and discrete spectral analysis, in: E. Quak, T. Soomere (eds.), Applied Wave Mathematics, Springer (2009) 301 -333. 